\section{Real-time national survey of COVID-19 in hemoglobinopathy and rare inherited anemia patients}

Faced with the rapidly evolving COVID-19 pandemic, in March 2020 the UK Government advocated strict self-isolation ('shielding') to protect extremely vulnerable patient groups deemed at high risk of severe SARS-CoV-2 infection. ${ }^{1}$ These included children and adults with sickle cell anemia (HbSS). On the advice of the National Hemoglobinopathy Panel (NHP), a multidisciplinary expert advisory group, shielding guidance was extended to all sickle cell disease (SCD) sub-types. Patients with transfusion dependent (TDT) and non-transfusion dependent thalassemia (NTDT), Diamond-Blackfan anemia (DBA) and other rare inherited anemias were also advised to shield if considered at high risk based on agreed clinical criteria. These included severe iron overload, splenectomy, diabetes and cardiac disease. ${ }^{2}$ Data provided by two participating centers with the largest thalassemia cohorts indicate up to $30 \%$ of patients meet these criteria.

In order to evaluate the impact of these measures and inform guidance on the clinical management of COVID-19 and public health policy, a real-time survey of confirmed and suspected cases of COVID-19 in hemoglobinopathy and rare inherited anemia patients was initiated on behalf of the NHP and National Health Service (NHS) England Clinical Reference Group for Hemoglobinopathies.

Data were submitted weekly by the 14 Hemoglobinopathy Coordinating Centers (HCC) in England, providing national coverage. HCC were encouraged to follow World Health Organization (WHO) case definitions which include both confirmed and clinically suspected COVID-19. ${ }^{3}$ Anonymised data were collected using a standardised report template (see the Online Supplementary Data) and presented weekly to the NHP. Between April 8 and May 6, 2020, a total of 195 confirmed or suspected COVID-19 cases (male: 87; female: 108) were reported. The timeline of case accrual is shown in Figure 1A. The median age was 33 years (range: 6 weeks to 92 years). The distribution according to age and sex is shown in Figure 1B. PCR for SARS-CoV-2 RNA was positive in 99 of $157(63 \%)$ cases tested (Figure $2 \mathrm{~A})$. Laboratory confirmation was not available for $34(17.4 \%)$ cases, 31 of which were managed in the community for suspected COVID-19 before widespread testing became available.

SCD accounted for 166 (85.1\%) of cases reported, with $129(77.7 \%)$ severe ( $\mathrm{HbSS}$ or $\mathrm{HbS} \beta^{\circ}$-thalassemia) and 37 $(22.3 \%)$ mild ( $\mathrm{HbSC}, \mathrm{HbS} \beta^{+}$-thalassemia or $\mathrm{HbSE}$ ) genotypes (Figures 2A-B). There were 149 adults and 17 children (defined as $\leq 18$ years). Ninety-five $(57 \%)$ were female. One hundred and twenty-eight (77.1\%) SCD patients were admitted to hospital of whom 15 (11.7\%), all adults, required non-invasive and/or mechanical ventilation (Figure $2 \mathrm{~B}$ ). The proportion of patients who required critical care was higher in mild genotypes, 8 of $29(27.6 \%)$, than severe genotypes, 7 of 99 (7.1\%) (Figure 2B). Sixty of $154(39 \%)$ patients for whom data were available received transfusion (red cell exchange 46 [29\%]; simple [top-up] transfusion 15 [10\%]) during the COVID-19 episode. The proportion of transfused patients was similar for children and adults. Outcome was analyzed for cases with a completed COVID-19 course ( $n=142)$, excluding those with a continuing inpatient stay or missing data. Of patients with a completed course, $131(92.2 \%)$ have recovered and 11 $(8.4 \%)$ died. The median age of patients who died was higher (51 years, range: 19-68 years) than those who
Table 1. Association of patient variables with survival status in sickle cell disease patients with laboratory confirmed COVID-19 admitted to hospital and outcome according to RT-PCR status

\begin{tabular}{lccc} 
& Alive & Dead & $P$ \\
Patient sex & & & \\
Female & $44(92 \%)$ & $4(8 \%)$ & 0.94 \\
$\quad$ Male & $25(86 \%)$ & $4(14 \%)$ & \\
Patient age (years) & & & \\
$<10$ & $1(100 \%)$ & $0(0 \%)$ & \\
$10-19.9$ & $8(89 \%)$ & $1(11 \%)$ & \\
$20-29.9$ & $24(92 \%)$ & $2(8 \%)$ & 0.14 \\
$30-39.9$ & $14(93 \%)$ & $1(7 \%)$ & \\
$40-49.9$ & $5(100 \%)$ & $0(0 \%)$ & \\
$50-59.9$ & $13(93 \%)$ & $1(0 \%)$ & \\
$>59.9$ & $4(57 \%)$ & $3(43 \%)$ & \\
\hline Disease severity & & & \\
Mild & $19(86 \%)$ & $3(14 \%)$ & 0.55 \\
Severe & $50(91 \%)$ & $5(9 \%)$ & \\
PCR status & & & \\
Negative & $47(98 \%)$ & $1(2 \%)$ & 0.081 \\
Positive & $69(90 \%)$ & $8(10 \%)$ & \\
\hline
\end{tabular}

recovered (31 years, range: 6 weeks to 72 years, $P=0.0042)$. No deaths occurred in children. Among adult patients admitted to hospital mortality was $9.2 \%$ (10 of 109). One patient died outside hospital, in a community residential care facility. In six patients who died, comorbid conditions (stroke: 1, cardiopulmonary disease: 2; cancer: 1; chronic kidney disease (stage G5): 1; hypertension: 1) associated with increased risk of death in COVID-19 were present. Seven of 10 patients who required mechanical ventilation died. As of May 6, 2020, 19 patients were receiving inpatient treatment, including non-invasive ventilation in one case. Data were missing for five patients managed outside hospital.

The association of patient variables with survival status was analyzed in SCD patients $(n=77)$ with a laboratory confirmed COVID-19 diagnosis who were admitted to hospital (Table 1). The overall mortality in the group was $10.4 \%$. In contrast to the association of older age and male sex with risk of COVID-19 related death in other populations, no significant correlation was found with age, sex or SCD genotype. Mortality was higher in females and mild genotypes though the differences did not reach significance.

As of May 6, 2020, 206,715 cases of COVID-19 with $30,615(14.8 \%)$ deaths had been reported in the UK. At first sight this suggests SCD patients are not more vulnerable to COVID-19. The age demographic profile of COVID-19 however differs markedly in the SCD and general population. In the ISARIC study ${ }^{4}$ of 20,133 cases of confirmed COVID-19 admitted to hospitals in the UK between February 6 and April 19, 2020, the median age was 73 years compared with a median age of 31 years in our cohort. We therefore compared the age adjusted risk of COVID-19 related death in SCD with that of the general population combining contemporaneous data from our survey, the National Hemoglobinopathy Registry (NHR $)^{5}$ and OpenSAFELY study. ${ }^{6}$ The latter (data cut May 6) quantified risk factors for COVID-19 related death in England by linking primary care electronic health records of 17 million patients with data from the COVID-19 National 
A

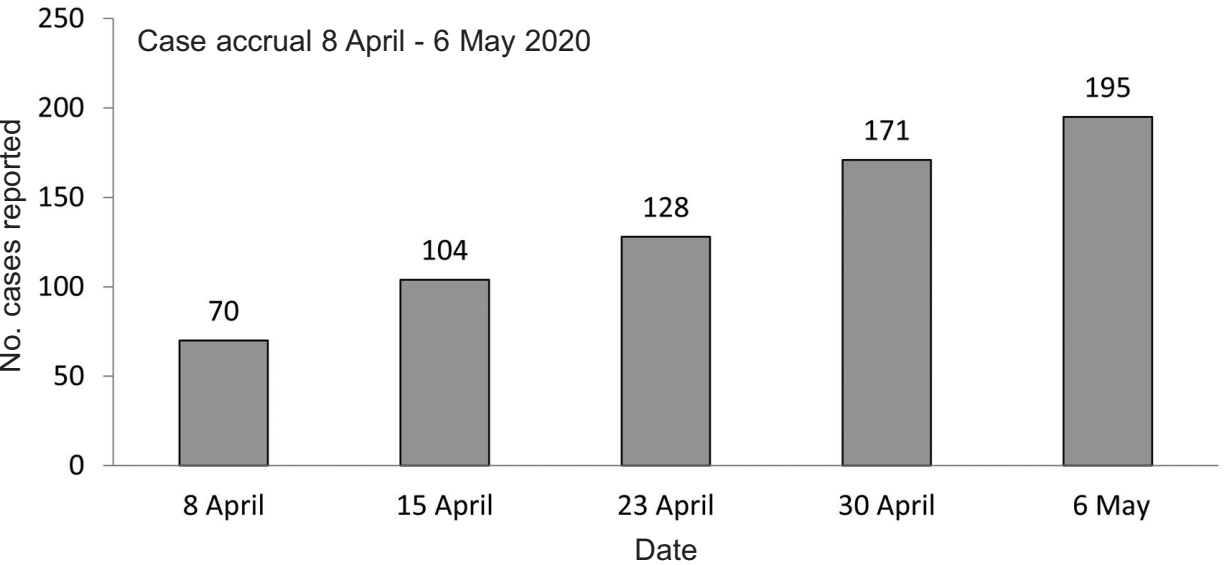

B

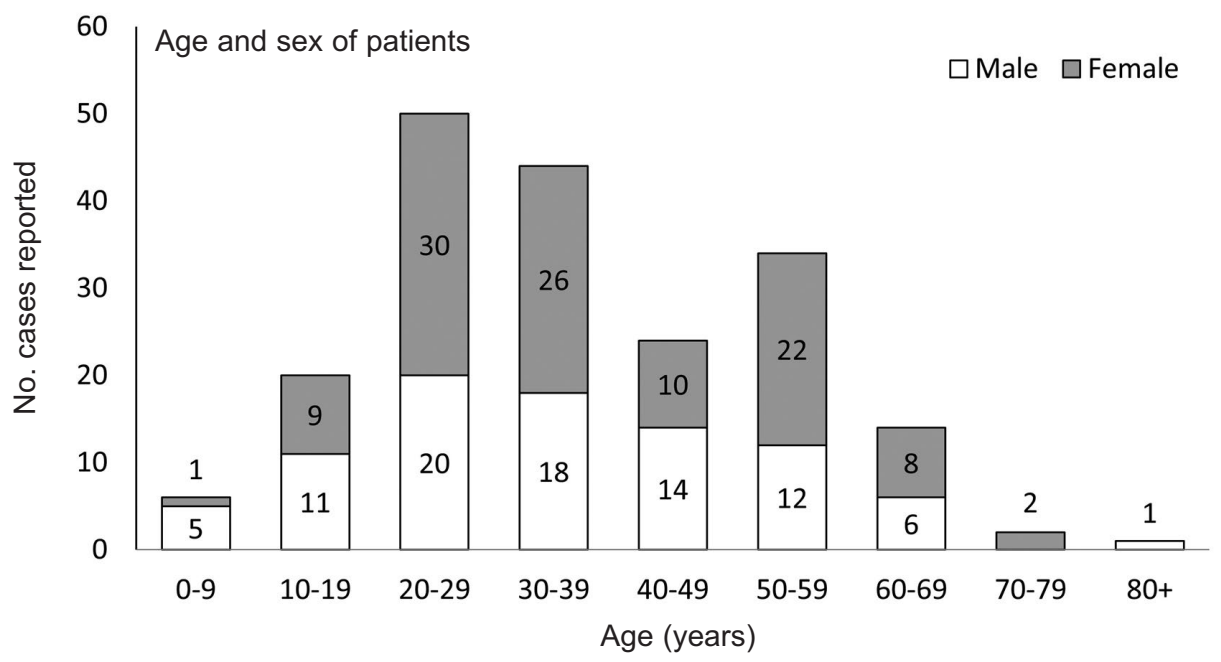

Figure 1. Number of confirmed and suspected COVID-19 cases reported over a 4-week period from April 8 to May 6, 2020 (A) subdivided according to age and $\operatorname{sex}(B)$.

Patient Reporting System. In this large cohort the COVID19 related mortality rate in patients aged $18-49$ years and $50-79$ years was 194 of 8,764,368 (22.1 per million people) and 4,258 of 7,382,344 (576 per million people) respectively. Based on the number of patients registered on the NHR in the same age groups, equivalent figures for SCD are 4 of 5,242 (763 per million people) and 5of 1,682 (2,972 per million people) respectively. Cases without a laboratory confirmed COVID-19 diagnosis were excluded from the analysis. Comparison of the proportions of deaths in SCD and the general population indicates that SCD patients in the age groups 18-49 years and 50-79 years have an increased risk of COVID-19 related death, odds ratio (OR) 34.5 (range: $12.8-92.8, P<0.0001$ ) and OR 4.1 (range: $1.5-$ $11.0, P=0.0047$ ) respectively.

Patient characteristics and outcomes for thalassemia $(n=26)$ are shown in Figure 2A and C. During the COVID19 episode $8(31 \%)$ thalassemia patients received transfusion. Two deaths were reported, in patients with hemoglobin $\mathrm{H}$ disease and TDT aged 92 and 53 years. Both had concurrent morbidities in the form of cancer and splenectomy, iron overload and diabetes respectively. Only three patients with rare inherited anemia (unstable hemoglobin Hb Köln: 2, hereditary elliptocytosis: 1) were reported (Figure 2A). All received transfusion for acute hemolysis during the COVID-19 episode and subsequently recovered. No cases of COVID-19 were reported in DBA.

As part of the survey HCC were encouraged to notify adverse events impacted by, though not necessarily directly attributable to COVID-19. Two deaths were reported in SCD, in which delayed presentation may have contributed to the outcome. In one case there was confirmed gramnegative sepsis and in the other SARS-CoV-2 RNA was detected post-mortem.

Despite guidance to reduce the risk of SARS-CoV-2 infection in extremely vulnerable individuals by strict selfisolation (shielding) at home and government support with food and medicines, a significant number of hemoglobinopathy patients in the UK have developed COVID19. It is likely that the reasons for this are complex. Identification of extremely vulnerable patients initially relied on national digital health data systems. In a survey conducted by one of the participating centers around $20 \%$ of patients reported they had not received the initial shielding guidance issued by the NHS. Socioeconomic factors such as low income and a need to preserve employment, poor housing, and lack of practical support limit the ability of some households to sustain effective shielding measures. Furthermore, nosocomial SARS-CoV-2 infection is a risk for patients who have to maintain contact with 


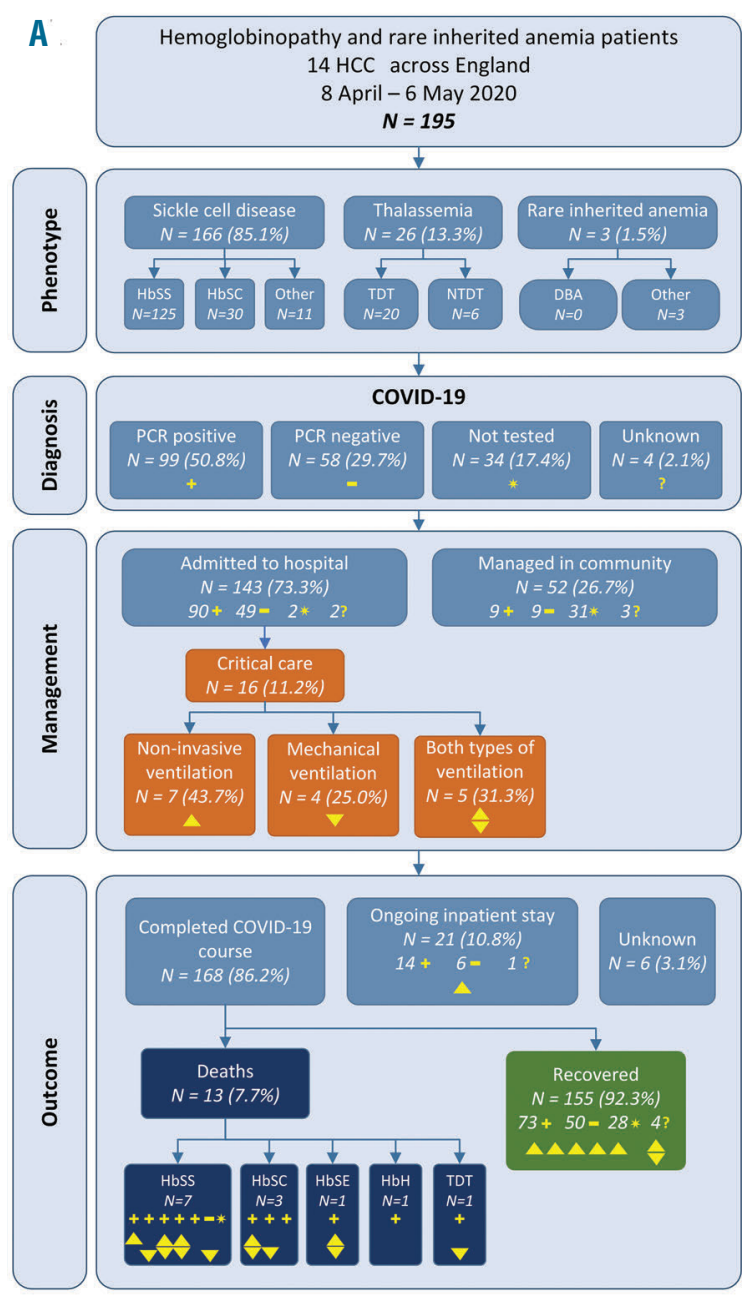

C

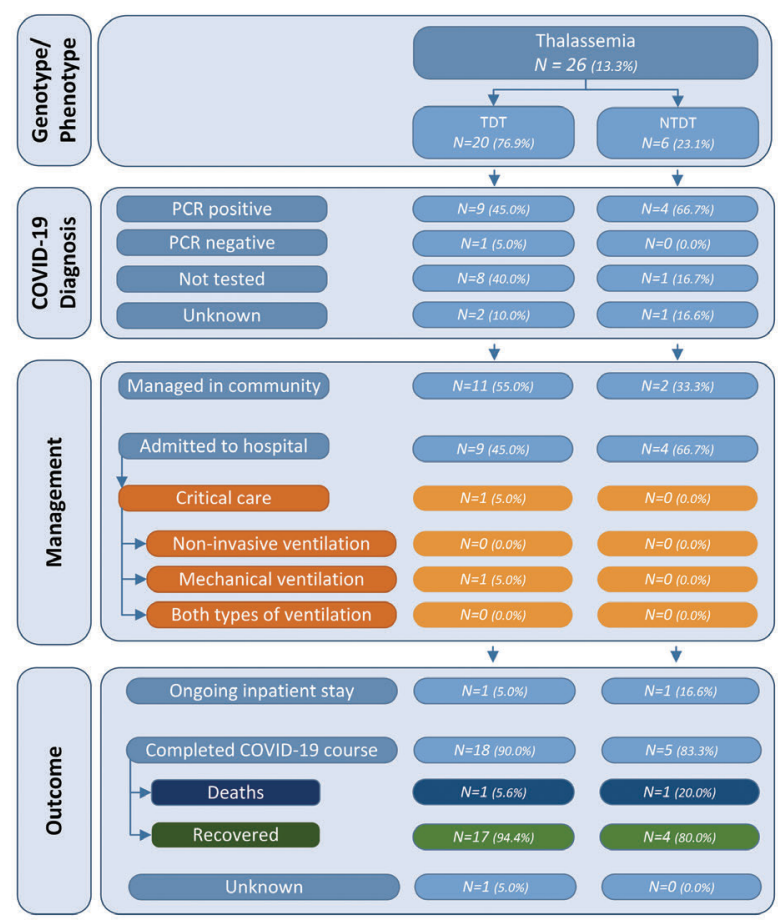

B

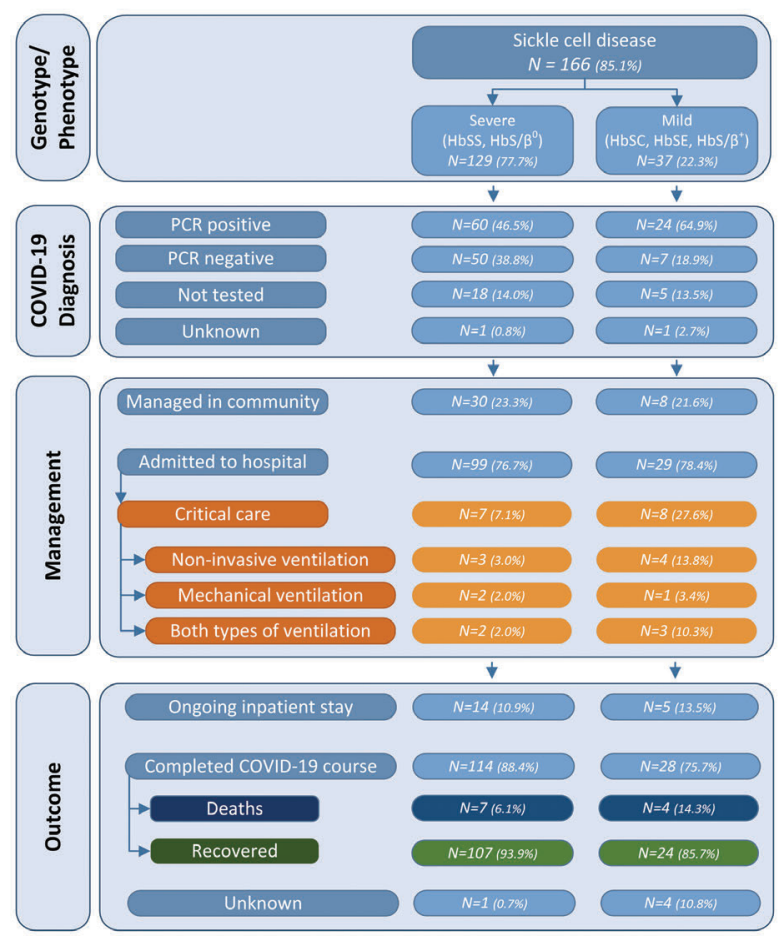

Figure 2. Schematic of the genotype/phenotype, COVID-19 diagnosis, management and outcome for patients with hemoglobinopathy or rare inherited anemia reported between April 8 and May 6, 2020. Total population (A), sickle cell disease (B) and thalassemia (C). TDT: transfusion dependent thalassemia; NTDT: non-transfusion dependent thalassemia; $\mathrm{HbH}$ : hemoglobin $\mathrm{H}$ disease. Symbols in yellow denote the PCR status and management of patients as follows: + PCR-positive; - PCR-negative; *: not tested; ?: status of testing unknown; triangle: non-invasive ventilation; inverted triangle: mechanical ventilation. For deaths, each column of symbols corresponds to an individual patient.

health care services for essential treatment. Our survey provides insight into the prevalence of COVID-19 in hemoglobinopathy and rare inherited anemia patients. It is estimated that there are 13,655 patients with SCD in England. ${ }^{7}$ Based on the age distribution of patients on the $\mathrm{NHR}$, cases reported represent $1.2 \%$ of the SCD patient population, comprising $1.8 \%$ of adults and $0.3 \%$ of children. The equivalent figures for thalassemia (with 1,564 patients on the NHR) are similar; overall $1.6 \%$, adults $2.3 \%$ and children $0.4 \%$. Nationally $35-40 \%$ of hemoglobinopathy patients fall into the pediatric age group. The low incidence of COVID-19 repor-ted in children with hemoglobinopathies is consistent with its characteristic course, which, with the exception of the hyperinflammatory syndrome described, ${ }^{8}$ is generally mild in this age group. This implies shielding may not be necessary for children with sickle cell and thalassemia disorders, other than in defined risk categories such as after hematopoietic stem cell transplantation. This has important implications for schooling and social development.

Two recent reports from France and the USA have described patient characteristics and outcomes of confirmed COVID-19 in SCD. ${ }^{9,10}$ In the USA registry hospitalization, intensive care unit (ICU) admission and case fatality rates were broadly similar to those seen in the UK. A higher rate of ICU admission (20.5\%) and lower mortality $(2.4 \%)$ was found among hospitalized patients in France. 
Consistent with our findings both studies observed a disproportionate number of COVID-19 rela-ted deaths in mild SCD genotypes. Differing conclusions were drawn with respect to overall morbidity and mortality of COVID19 in SCD. This highlights the importance of populationbased risk estimates which neither study included. In this regard the preliminary results reported here suggest COVID-19 is associated with increased mortality in adults with SCD. Further studies are needed to refine the magnitude of risk and determine the extent to which it reflects an independent effect or association with other clinical, demographic or socioeconomic risk factors. Current evidence suggests patients with sickle-related chronic organ damage may be at increased risk irrespective of age and underpins a ratio-nale for individual risk assessment in shielding guidance. This has important implications for prevention as European countries proceed with a phased easing of lockdown measures in the population.

In thalassemia and rare anemias, only tentative conclusions can be drawn given the small number of cases reported. In the former, outcomes were in keeping with an initial report from Italy, which indicates the course of COVID-19 in thalassemia is generally favorable. ${ }^{11}$ Both thalassemia cases associated with a fatal outcome in our survey had independent predictors of mortality.

HCC were instructed to notify both proven and clinically suspected cases of COVID-19, in contrast to the registry studies reported. In the early stages of the pandemic, laboratory capacity in the UK was limited and many patients with clinical features of COVID-19, particularly those with milder disease, were not tested. The limited sensitivity of RT-PCR testing for SARS-CoV-2 infection, with false negative rates up to $30 \%,{ }^{12}$ was a further consideration in our decision to include clinically suspected cases. In order to avoid ascertainment bias the use of WHO criteria for suspected cases was recommended. Nevertheless, this may have led to an overestimation of cases which, with the availability of testing for antibodies to SARS-CoV-2, it will be possible to evaluate retrospectively. Although all HCC were invited to participate not all returned complete data due to workforce constraints or competing priorities during the pandemic. HCC have been encouraged to retrospectively enter data. This will enable a complete analysis to be reported in due course, allowing estimates of incidence and morbidity to be refined.

Although not its primary purpose, our survey raises concern that an unintended consequence of shielding could be the delayed presentation of life-threatening complications in hemoglobinopathy and rare anemia patients. Observational studies to measure excess deaths in vulnerable patient groups during the COVID-19 pandemic are needed. In order to mitigate this risk the NHP in collaboration with NHS England and patient support groups issued guidance that while shielding patients should continue to access normal pathways for managing complications of their condition and notify their center of care immediately if they develop suspected COVID-19 symptoms to ensure presentations which overlap, inclu-ding bacterial infection and acute chest syndrome, are recognized and treated promptly. ${ }^{2}$ Our national survey, undertaken in a real-world setting, expands on case series from single centers ${ }^{13,14}$ and complements recently repor-ted registry data from France and the USA in contributing to an understanding of the direct and collateral impact of COVID-19 in SCD and other inherited anemias.

Paul Telfer, Josu de la Fuente, 2,3 Mamta Sohal, ${ }^{2}$ Ralph Brown, ${ }^{2}$ Perla Eleftheriou, ${ }^{4}$ Noémi Roy,
Frédéric B. Piel, 7 Subarna Chakravorty, Kate Gardner, Mark Velangi, ${ }^{10}$ Emma Drasar, ${ }^{4,11}$ Farrukh Shah,, 11 John B. Porter, ${ }^{4}$ Sara Trompeter, ${ }^{4,12}$ Wale Atoyebi, Richard Szydlo, ${ }^{13}$ Kofi A. Anie, ${ }^{14}$ Kate Ryan, ${ }^{15}$ Joseph Sharif, 15 Josh Wright, ${ }^{16}$ Emma Astwood, ${ }^{17}$ C. Sarah Nicolle, ${ }^{18}$ Amy Webster, ${ }^{18}$ David J. Roberts, ${ }^{5,6,20}$ Sanne Lugthart, ${ }^{21}$ Banu Kaya, Moji Awogbade, ${ }^{8}$ David C. Rees, Rob Hollingsworth, ${ }^{22}$ Baba Inusa, Jo Howard" and D. Mark Layton ${ }^{2,3}$ for the Hemoglobinopathy Coordinating Centers and National Hemoglobinopathy Panel, England

${ }^{\prime} B$ arts Health NHS Trust, London; 'Imperial College Healthcare NHS Trust, London; ${ }^{3}$ NIHR Imperial Biomedical Research Centre, London; ${ }^{4}$ University College London Hospitals NHS Foundation Trust, London; 50 hn Radcliffe Hospital, Oxford; ${ }^{6}$ NIHR Biomedical Research Centre, Oxford; 'School of Public Health, Faculty of Medicine, Imperial College London, London; ${ }^{8}$ King's College Hospital NHS Foundation Trust, London; 'Guy's and St Thomas' NHS Foundation Trust, London; ${ }^{10}$ Birmingham Children's Hospital, Birmingham; "Whittington Health NHS Trust, London; ${ }^{12}$ NHS Blood and Transplant, London; ${ }^{13}$ Imperial College, London; ${ }^{14}$ London North West University Healthcare NHS Trust, London; ${ }^{15}$ Manchester University NHS Foundation Trust, Manchester; ${ }^{16}$ Sheffield Teaching Hospital, Sheffield; "'Sheffield Children's Hospital, Sheffield;

${ }^{18}$ University Hospitals Coventry and Warwickshire NHS Trust, Coventry; ${ }^{19}$ University Hospitals of Leicester, Leicester; ${ }^{20}$ NHS Blood and Transplant, Oxford; "21 University Hospitals Bristol, Bristol, UK and ${ }^{22}$ Medical Data Solutions and Services, Manchester, UK

Correspondence:

MARK LAYTON -m.layton@imperial.ac.uk doi:10.3324/haematol.2020.259440

\section{References}

1. https://www.gov.uk/government/publications/guidance-on-shielding-and-protecting-extremely-vulnerable-persons-from-covid19/guidance-on-shielding-and-protecting-extremely-vulnerable-persons-from-covid-19

2. https://b-s-h.org.uk/media/18244/hbp-hccs-response-to-covid-v9200420.pdf

3. World Health Organization.( 2020). Global surveillance for COVID19 caused by human infection with COVID-19 virus: interim guidance, 20 March 2020

4. Docherty AB, Harrison EM, Green CA, et al. Features of 20,133 UK patients in hospital with covid-19 using the ISARIC WHO Clinical Characterisation Protocol: prospective observational cohort study. BMJ. 2020;369:m1985

5. http://nhr.mdsas.com

6. Williamson EJ, Walker AJ, Bhaskaran K, et al. Factors associated with COVID-19-related death using OpenSAFELY. Nature. 2020;584(7821):430-436.

7. Dormandy E, James J, Inusa B, Rees D. How many people have sickle cell disease in the UK? J Public Health (Oxf). 2018;40(3):e291e295.

8. Riphagen S, Gomez X, Gonzalez-Martinez C, Wilkinson N Theocharis P. Hyperinflammatory shock in children during COVID19 pandemic. Lancet. 2020;395(10237):1607-1608.

9. Arlet JB, de Luna G, Khimoud D, et al. Prognosis of patients with sickle cell disease and COVID-19: a French experience. Lancet Haematol. 2020;7(9):e632-e634

10. Panepinto JA, Brandow A, Mucalo L, et al. Coronavirus disease among persons with sickle cell disease, United States, March 20 May 21, 2020. Emerg Infect Dis. 2020;26(10).

11. Motta I, Migone De Amicis M, et al. SARS-CoV-2 infection in beta thalassemia: Preliminary data from the Italian experience. Am J Hematol. 2020;95(8):E198-E199.

12. Woloshin S, Patel N, Kesselheim AS. False negative tests for SARS CoV-2 infection - challenges and implications. N Engl J Med. 2020; 383(6).e38.

13. McCloskey KA, Meenan J, Hall R, Tsitsikas DA. COVID-19 Infection and sickle cell disease: a UK centre experience. $\mathrm{Br}$ J Haematol. 2020;190(2):e57-e58.

14. Hussain FA, Njoku FU, Saraf SL, Molokie RE, Gordeuk VR, Han J. COVID-19 infection in patients with sickle cell disease. $\mathrm{Br}$ J Haematol. 2020;189(5):851-852 